\title{
The New Formulas for the Eigenvectors of the Gaudin Model in the so(5) Case.
}

\author{
Č. Burdík†and O. Navrátilł \\ $\dagger$ Department of Mathematics, Czech Technical University in Prague, Faculty of \\ Nuclear Sciences and Physical Engineering, Trojanova 13, 12000 Prague 2, Czech \\ Republic \\ $\ddagger$ Department of Mathematics, Czech Technical University in Prague, Faculty of \\ Transportation Sciences, Na Florenci 25, 11000 Prague, Czech Republic
}

\begin{abstract}
In our paper [1] we proposed new formulas for eigenvectors of he Gaudin model in sl(3) case. Similarly in this paper we used the standard Bethe Ansatz method for finding the eigenvectors and the eigenvalues in the so(5) case in an explicit form.
\end{abstract}

PACS numbers: 0365F, 0220S 


\section{Introduction}

In 1973 M. Gaudin [2-4], proposed a new class of integrable quantum models. This sl(2) Gaudin model was studied by many authors [2-11] from different point of view. Let us remember you well know Bethe Ansatz method for finding the eigenvectors and the eigenvalues $[3,12-14]$ in this case.

Let the generators $e, f$ and $h$ form a standard basis in $\operatorname{sl}(2)$ which fulfil the commutations relations

$$
[h, e]=2 e, \quad[h, f]=-2 f, \quad[e, f]=h .
$$

For second order Casimir operator we obtain

$$
C=e f+f e+\frac{1}{2} h^{2} .
$$

We will define

$$
F(u)=\sum_{i=1}^{N} \frac{f^{(i)}}{u-z_{i}}, \quad E(u)=\sum_{i=1}^{N} \frac{e^{(i)}}{u-z_{i}}, \quad H(u)=\sum_{i=1}^{N} \frac{h^{(i)}}{u-z_{i}} .
$$

The central point in Bethe ansatz method for Gaudin model play an operator

$$
T(u)=\frac{1}{2}\left(E(u) F(u)+F(u) E(u)+\frac{1}{2} H^{2}(u)\right) .
$$

It is possible to rewrite the operator (3) in the form

$$
T(u)=\sum_{i=1}^{N} \frac{H_{i}}{u-z_{i}}+\frac{1}{2} \sum_{i=1}^{N} \frac{C^{(i)}}{\left(u-z_{i}\right)^{2}},
$$

where $H_{i}$ are given by

$$
H_{i}=\sum_{j \neq i} \frac{\frac{1}{2} h^{(i)} h^{(j)}+e^{(i)} f^{(j)}+e^{(j)} f^{(i)}}{z_{i}-z_{j}}
$$

and $C^{(i)}$ is the Casimir operator acting on the $i^{\text {th }}$ factor of $V_{(\lambda)}$.

It is easy calculation to show that from the commutation relations (1) and the definitions (2) we obtain for $u \neq w$

$$
\begin{aligned}
{[E(u), E(w)] } & =[F(u), F(w)]=[H(u), H(w)]=0, \\
{[E(u), F(w)] } & =-\frac{H(u)-H(w)}{u-w}, \\
{[H(u), E(w)] } & =-2 \frac{E(u)-E(w)}{u-w}, \\
{[H(u), F(w)] } & =2 \frac{F(u)-F(w)}{u-w} .
\end{aligned}
$$

For construction of Gaudin model we will use highest representations

$$
e^{(i)} v_{\lambda^{(i)}}=0, \quad h^{(i)} v_{\lambda^{(i)}}=\lambda^{(i)} v_{\lambda^{(i)}}
$$


The New Formulas for the Eigenvectors of the Gaudin Model in the so(5) Case.

It is easy to see that the vector $|0\rangle=v_{\lambda^{(1)}} \otimes v_{\lambda^{(2)}} \otimes \ldots \otimes v_{\lambda^{(N)}}$ is eigenvectors of $T(u)$ and the relations

$$
E(u)|0\rangle=0, \quad H(u)|0\rangle=\lambda(u)|0\rangle=\sum_{i=1}^{N} \frac{\lambda^{(i)}}{u-z_{i}}|0\rangle, \quad T(u)|0\rangle=\tau(u)|0\rangle,
$$

where $\tau(u)=\frac{1}{4} \lambda^{2}(u)-\frac{1}{2} \lambda^{\prime}(u)$, are hold.

We will fix notation

$$
\begin{aligned}
& F(\mathbf{w})=F\left(w_{1}\right) F\left(w_{2}\right) \ldots F\left(w_{n}\right), \\
& F\left(\mathbf{w}-w_{r}\right)=F\left(w_{1}\right) \ldots F\left(w_{r-1}\right) F\left(w_{r+1}\right) \ldots F\left(w_{n}\right), \\
& F(\mathbf{w}+u)=F(u) F\left(w_{1}\right) \ldots F\left(w_{n}\right)
\end{aligned}
$$

and we can try to obtain in correspondence with Bethe Ansatz method further eigenvalues as $|\mathbf{w}\rangle=F(\mathbf{w})|0\rangle$.

Direct calculation gives

$$
\begin{aligned}
{[T(u), F(\mathbf{w})]=} & -\sum_{r=1}^{n} \frac{F(\mathbf{w})}{u-w_{r}}\left(H(u)-\sum_{s \neq r} \frac{1}{u-w_{s}}\right)+ \\
& +\sum_{r=1}^{n} \frac{F\left(\mathbf{w}+u-w_{r}\right)}{u-w_{r}}\left(H\left(w_{r}\right)-\sum_{s \neq r} \frac{2}{w_{r}-w_{s}}\right) .
\end{aligned}
$$

Applying this equation to the hight vector $|0\rangle$ we obtain

$$
T(u)|\mathbf{w}\rangle=T_{0}(u)|\mathbf{w}\rangle+T_{1}(u)|\mathbf{w}\rangle,
$$

where

$$
\begin{aligned}
& T_{0}(u)|\mathbf{w}\rangle=\tau(u)|\mathbf{w}\rangle-\sum_{r=1}^{n}\left(\lambda(u)-\sum_{s \neq r} \frac{1}{u-w_{s}}\right) \frac{|\mathbf{w}\rangle}{u-w_{r}}, \\
& T_{1}(u)|\mathbf{w}\rangle=\sum_{r=1}^{n}\left(\lambda\left(w_{r}\right)-\sum_{s \neq r} \frac{2}{w_{r}-w_{s}}\right) \frac{\left|\mathbf{w}+u-w_{r}\right\rangle}{u-w_{r}} .
\end{aligned}
$$

It is evident that $|\mathbf{w}\rangle$ is eigenvector $T(u)$ for all $u$ if

$$
T_{1}(u)|\mathbf{w}\rangle=0 \quad \text { and } \quad T_{0}(u)|\mathbf{w}\rangle=\tau(u ; \mathbf{w})|\mathbf{w}\rangle .
$$

The first equation is equivalent to the Bethe equations

$$
\lambda\left(w_{r}\right)-\sum_{s \neq r} \frac{2}{w_{r}-w_{s}}=0 \text { for all } r=1, \ldots, n
$$

and the second condition gives corresponding eigenvalue

$$
\tau(u ; \mathbf{w})=\tau(u)-\sum_{r=1}^{n} \frac{\lambda(u)}{u-w_{r}}+\sum_{r \neq s} \frac{2}{\left(u-w_{r}\right)\left(w_{r}-w_{s}\right)} .
$$


The New Formulas for the Eigenvectors of the Gaudin Model in the so(5) Case.

\section{The Gaudin model for so(5).}

Now we will similarly as in the case sl(3) [1] use the Bethe ansatz method for so(5) model. This algebra is 10-dimensional and we will use as a basis $\mathbf{h}_{1}, \mathbf{h}_{2}, \mathbf{e}_{k}$ a $\mathbf{f}_{k}, k=1, \ldots, 4$. The second order Casimir operator is given by formula

$$
\mathbf{C}_{2}=\sum_{k=1}^{4}\left(\mathbf{e}_{k} \mathbf{f}_{k}+\mathbf{f}_{k} \mathbf{e}_{k}\right)+\mathbf{h}_{1}^{2}+\mathbf{h}_{2}^{2}
$$

We define by $X(u)=\sum_{i=1}^{N} \frac{\mathbf{x}^{(i)}}{u-z_{i}}$ for any element of basis and we have

$$
[X(u), Y(w)]=-\frac{Z(u)-Z(w)}{u-w} \quad \text { iff }[\mathbf{x}, \mathbf{y}]=\mathbf{z} .
$$

For operator $T(u)$ we obtain

$$
\begin{aligned}
T(u) & =\frac{1}{2}\left(\sum_{k=1}^{4}\left(E_{k}(u) F_{k}(u)+F_{k}(u) E_{k}(u)\right)+H_{1}^{2}(u)+H_{2}^{2}(u)\right)= \\
& =\sum_{k=1}^{4} F_{k}(u) E_{k}(u)+\frac{1}{2}\left(H_{1}^{2}(u)+H_{2}^{2}(u)-3 H_{1}^{\prime}(u)-H_{2}^{\prime}(u)\right) .
\end{aligned}
$$

For any $k=1, \ldots, 4$ we will define

$$
F_{k}\left(\mathbf{w}_{\mathbf{k}}\right)=F\left(w_{k, 1}\right) F\left(w_{k, 2}\right) \ldots F\left(w_{k, n_{k}}\right)
$$

and because $F_{k}\left(\mathbf{w}_{\mathbf{k}}\right)$ not commute more we will fix ordering $F_{2}\left(\mathbf{w}_{\mathbf{2}}\right), F_{3}\left(\mathbf{w}_{\mathbf{3}}\right), F_{4}\left(\mathbf{w}_{\mathbf{4}}\right)$, $F_{1}\left(\mathbf{w}_{\mathbf{1}}\right)$. We know from paper [15] that generally it is not possible to find the eigenvalues in the form

$$
\left|\mathbf{w}_{2}, \mathbf{w}_{3}, \mathbf{w}_{4}, \mathbf{w}_{1}\right\rangle=F_{2}\left(\mathbf{w}_{\mathbf{2}}\right) F_{3}\left(\mathbf{w}_{\mathbf{3}}\right) F_{4}\left(\mathbf{w}_{\mathbf{4}}\right) F_{1}\left(\mathbf{w}_{\mathbf{1}}\right)|0\rangle
$$

but anyway we finally obtain explicit form of action $T(u)$ on such vectors. (see Appendix B for explicit formulas). In this representation we start with

$$
E_{k}(u)|0\rangle=0, \quad H_{i}(u)|0\rangle=\lambda_{i}(u)|0\rangle, \quad T(u)|0\rangle=\tau(u)|0\rangle,
$$

where $\tau(u)=\frac{1}{2}\left(\lambda_{1}^{2}(u)+\lambda_{2}^{2}(u)-3 \lambda_{1}^{\prime}(u)-\lambda_{2}^{\prime}(u)\right)$.

For the calculation will be useful next lemmas.

Lemma 1. If we denote $H_{ \pm}(u)=H_{1}(u) \pm H_{2}(u)$, we obtain

$$
\begin{aligned}
{\left[T(u), F_{1}(w)\right]=} & \frac{F_{1}(u) H_{2}(w)-F_{1}(w) H_{2}(u)}{u-w}+\frac{F_{3}(w) E_{2}(u)-F_{3}(u) E_{2}(w)}{u-w}+ \\
& +\frac{F_{4}(w) E_{3}(u)-F_{4}(u) E_{3}(w)}{u-w} \\
{\left[T(u), F_{2}(w)\right]=} & \frac{F_{2}(u) H_{-}(w)-F_{2}(w) H_{-}(u)}{u-w}+\frac{F_{3}(u) E_{1}(w)-F_{3}(w) E_{1}(u)}{u-w} \\
{\left[T(u), F_{3}(w)\right]=} & \frac{F_{2}(u) F_{1}(w)-F_{2}(w) F_{1}(u)}{u-w}+\frac{F_{3}(u) H_{1}(w)-F_{3}(w) H_{1}(u)}{u-w}+ \\
& +\frac{F_{4}(u) E_{1}(w)-F_{4}(w) E_{1}(u)}{u-w} \\
{\left[T(u), F_{4}(w)\right]=} & \frac{F_{3}(u) F_{1}(w)-F_{3}(w) F_{1}(u)}{u-w}+\frac{F_{4}(u) H_{+}(w)-F_{4}(w) H_{+}(u)}{u-w} .
\end{aligned}
$$


Proof: The simple direct calculation.

We will fix again notation

$$
\begin{aligned}
& F_{k}\left(\mathbf{w}_{\mathbf{k}}\right)=F_{k}\left(w_{k, 1}\right) F_{k}\left(w_{k, 2}\right) \ldots F_{k}\left(w_{k, n}\right) \\
& F_{k}\left(\mathbf{w}_{\mathbf{k}}-w_{k, r}\right)=F\left(w_{k, 1}\right) \ldots F_{k}\left(w_{k, r-1}\right) F_{k}\left(w_{k, r+1}\right) \ldots F_{k}\left(w_{k, n}\right), \\
& F_{k}\left(\mathbf{w}_{\mathbf{k}}+u\right)=F_{k}(u) F_{k}\left(w_{k, 1}\right) \ldots F_{k}\left(w_{k, n}\right) .
\end{aligned}
$$

Lemma 2. For commutators $T(u)$ with $F_{1}\left(\mathbf{w}_{\mathbf{1}}\right), F_{2}\left(\mathbf{w}_{\mathbf{2}}\right), F_{3}\left(\mathbf{w}_{\mathbf{3}}\right), F_{4}\left(\mathbf{w}_{\mathbf{4}}\right)$ we obtain

$$
\begin{aligned}
& {\left[T(u), F_{1}\left(\mathbf{w}_{1}\right)\right]=\sum_{r} \frac{F_{1}\left(\mathbf{w}_{1}+u-w_{r}\right)}{u-w_{r}}\left(H_{2}\left(w_{r}\right)-\sum_{\widehat{r} \neq r} \frac{1}{w_{r}-w_{\widehat{r}}}\right)-} \\
& -\sum_{r} \frac{F_{1}\left(\mathbf{w}_{1}\right)}{u-w_{r}}\left(H_{2}(u)-\sum_{\widehat{r} \neq r} \frac{1}{w_{r}-w_{\widehat{r}}}\right)+ \\
& +\sum_{r} F_{3}\left(w_{r}\right) \frac{F_{1}\left(\mathbf{w}_{1}-w_{r}\right)}{u-w_{r}} E_{2}(u)-\sum_{r} F_{3}(u) \frac{F_{1}\left(\mathbf{w}_{1}-w_{r}\right)}{u-w_{r}} E_{2}\left(w_{r}\right)+ \\
& +\sum_{r} F_{4}\left(w_{r}\right) \frac{F_{1}\left(\mathbf{w}_{1}-w_{r}\right)}{u-w_{r}} E_{3}(u)-\sum_{r} F_{4}(u) \frac{F_{1}\left(\mathbf{w}_{1}-w_{r}\right)}{u-w_{r}} E_{3}\left(w_{r}\right)- \\
& -\sum_{r \neq \widehat{r}} F_{4}(u) \frac{F_{1}\left(\mathbf{w}_{1}-w_{r}-w_{\widehat{r}}\right)}{\left(u-w_{r}\right)\left(w_{r}-w_{\widehat{r}}\right)} E_{2}\left(w_{r}\right)+ \\
& +\sum_{r \neq \widehat{r}} F_{4}\left(w_{r}\right) \frac{F_{1}\left(\mathbf{w}_{1}-w_{r}-w_{\widehat{r}}\right)}{\left(u-w_{r}\right)\left(w_{r}-w_{\widehat{r}}\right)} E_{2}(u) \\
& {\left[T(u), F_{2}\left(\mathbf{w}_{2}\right)\right]=\sum_{s} \frac{F_{2}\left(\mathbf{w}_{2}+u-w_{s}\right)}{u-w_{s}}\left(H_{-}\left(w_{s}\right)-\sum_{\widehat{s} \neq s} \frac{2}{w_{s}-w_{\widehat{s}}}\right)-} \\
& -\sum_{s} \frac{F_{2}\left(\mathbf{w}_{2}\right)}{u-w_{s}}\left(H_{-}(u)-\sum_{\widehat{s} \neq s} \frac{2}{w_{s}-w_{\widehat{s}}}\right)+ \\
& +\sum_{s} \frac{F_{2}\left(\mathbf{w}_{2}-w_{s}\right)}{u-w_{s}}\left(F_{3}(u) E_{1}\left(w_{s}\right)-F_{3}\left(w_{s}\right) E_{1}(u)\right) \\
& {\left[T(u), F_{3}\left(\mathbf{w}_{3}\right]=\sum_{t} F_{2}(u) \frac{F_{3}\left(\mathbf{w}_{3}-w_{t}\right)}{u-w_{t}} F_{1}\left(w_{t}\right)-\sum_{t} F_{2}\left(w_{t}\right) \frac{F_{3}\left(\mathbf{w}_{3}-w_{t}\right)}{u-w_{t}} F_{1}(u)+\right.} \\
& +\sum_{t} \frac{F_{3}\left(\mathbf{w}_{3}+u-w_{t}\right)}{u-w_{t}}\left(H_{1}\left(w_{t}\right)-\sum_{\widehat{t} \neq t} \frac{1}{w_{t}-w_{\hat{t}}}\right)- \\
& -\sum_{t} \frac{F_{3}\left(\mathbf{w}_{3}\right)}{u-w_{t}}\left(H_{1}(u)-\sum_{\hat{t} \neq t} \frac{1}{w_{t}-w_{\hat{t}}}\right)+ \\
& +\sum_{t} \frac{F_{3}\left(\mathbf{w}_{3}-w_{t}\right)}{u-w_{t}}\left(F_{4}(u) E_{1}\left(w_{t}\right)-F_{4}\left(w_{t}\right) E_{1}(u)\right)- \\
& -\sum_{t \neq \hat{t}} F_{2}(u) \frac{F_{3}\left(\mathbf{w}_{3}-w_{t}-w_{\hat{t}}\right)}{\left(u-w_{\hat{t}}\right)\left(w_{\hat{t}}-w_{t}\right)} F_{4}\left(w_{t}\right)- \\
& -\sum_{t \neq \hat{t}} F_{2}\left(w_{t}\right) \frac{F_{3}\left(\mathbf{w}_{3}-w_{t}-w_{\hat{t}}\right)}{\left(u-w_{\hat{t}}\right)\left(w_{\hat{t}}-w_{t}\right)} F_{4}(u)+ \\
& +\sum_{t \neq \hat{t}} F_{2}\left(w_{t}\right) \frac{F_{3}\left(\mathbf{w}_{3}-w_{t}-w_{\hat{t}}\right)}{\left(u-w_{t}\right)\left(u-w_{\hat{t}}\right)} F_{4}\left(w_{\hat{t}}\right)
\end{aligned}
$$


The New Formulas for the Eigenvectors of the Gaudin Model in the so(5) Case.

$$
\begin{aligned}
{\left[T(u), F_{4}\left(\mathbf{w}_{4}\right)\right]=} & \sum_{r} F_{3}(u) \frac{F_{4}\left(\mathbf{w}_{4}-w_{r}\right)}{u-w_{r}} F_{1}\left(w_{r}\right)-\sum_{r} F_{3}\left(w_{r}\right) \frac{F_{4}\left(\mathbf{w}_{4}-w_{r}\right)}{u-w_{r}} F_{1}(u)+ \\
& +\sum_{r} \frac{F_{4}\left(\mathbf{w}_{4}+u-w_{r}\right)}{u-w_{r}}\left(H_{+}\left(w_{r}\right)-\sum_{\widehat{r} \neq r} \frac{2}{w_{r}-w_{\widehat{r}}}\right)- \\
& -\sum_{r} \frac{F_{4}\left(\mathbf{w}_{4}\right)}{u-w_{r}}\left(H_{+}(u)-\sum_{\widehat{r} \neq r} \frac{2}{w_{r}-w_{\widehat{r}}}\right)
\end{aligned}
$$

Now we can calculate the action of $T(u)$ by this way

$$
\begin{aligned}
T(u) F_{2}\left(\mathbf{w}_{\mathbf{2}}\right) F_{3}\left(\mathbf{w}_{\mathbf{3}}\right) F_{4}\left(\mathbf{w}_{\mathbf{4}}\right) F_{1}\left(\mathbf{w}_{\mathbf{1}}\right) & =F_{2}\left(\mathbf{w}_{\mathbf{2}}\right) F_{3}\left(\mathbf{w}_{\mathbf{3}}\right) F_{4}\left(\mathbf{w}_{\mathbf{4}}\right) F_{1}\left(\mathbf{w}_{\mathbf{1}}\right) T(u)+ \\
& +\left[T(u), F_{2}\left(\mathbf{w}_{\mathbf{2}}\right)\right] F_{3}\left(\mathbf{w}_{\mathbf{3}}\right) F_{4}\left(\mathbf{w}_{\mathbf{4}}\right) F_{1}\left(\mathbf{w}_{\mathbf{1}}\right)+ \\
& +F_{2}\left(\mathbf{w}_{\mathbf{2}}\right)\left[T(u), F_{3}\left(\mathbf{w}_{\mathbf{3}}\right)\right] F_{4}\left(\mathbf{w}_{\mathbf{4}}\right) F_{1}\left(\mathbf{w}_{\mathbf{1}}\right)+ \\
& +F_{2}\left(\mathbf{w}_{\mathbf{2}}\right) F_{3}\left(\mathbf{w}_{\mathbf{3}}\right)\left[T(u), F_{4}\left(\mathbf{w}_{\mathbf{4}}\right)\right] F_{1}\left(\mathbf{w}_{\mathbf{1}}\right)+ \\
& +F_{2}\left(\mathbf{w}_{\mathbf{2}}\right) F_{3}\left(\mathbf{w}_{\mathbf{3}}\right) F_{4}\left(\mathbf{w}_{\mathbf{4}}\right)\left[T(u), F_{1}\left(\mathbf{w}_{\mathbf{1}}\right)\right] .
\end{aligned}
$$

But we see that from lemma 2 that some generators are not still in good position. In the next lemma we summarize formulas for $F_{3}\left(\mathbf{w}_{\mathbf{3}}\right)$ case, which will be useful for reordering.

\section{Lemma 3.}

$$
\begin{aligned}
F_{1}(u) F_{3}\left(\mathbf{w}_{3}\right)= & F_{3}\left(\mathbf{w}_{3}\right) F_{1}(u)+\sum_{t} \frac{F_{3}\left(\mathbf{w}_{3}-w_{t}\right)}{u-w_{t}}\left(F_{4}(u)-F_{4}\left(w_{t}\right)\right) \\
F_{3}\left(\mathbf{w}_{3}\right) F_{2}(u)= & F_{2}(u) F_{3}\left(\mathbf{w}_{3}\right) \\
F_{3}(u) F_{3}\left(\mathbf{w}_{3}\right)= & F_{3}\left(\mathbf{w}_{3}+u\right) \\
F_{4}(u) F_{3}\left(\mathbf{w}_{3}\right)= & F_{3}\left(\mathbf{w}_{3}\right) F_{4}(u) \\
H_{1}(u) F_{3}\left(\mathbf{w}_{3}\right)= & F_{3}\left(\mathbf{w}_{3}\right)\left(H_{1}(u)-\sum_{t} \frac{1}{u-w_{t}}\right)+\sum_{t} \frac{F_{3}\left(\mathbf{w}_{3}+u-w_{t}\right)}{u-w_{t}} \\
H_{2}(u) F_{3}\left(\mathbf{w}_{3}\right)= & F_{3}\left(\mathbf{w}_{3}\right) H_{2}(u) \\
E_{1}(u) F_{3}\left(\mathbf{w}_{3}\right)= & F_{3}\left(\mathbf{w}_{3}\right) E_{1}(u)+\sum_{t}\left(F_{2}(u)-F_{2}\left(w_{t}\right)\right) \frac{F_{3}\left(\mathbf{w}_{3}-w_{t}\right)}{u-w_{t}} \\
E_{2}(u) F_{3}\left(\mathbf{w}_{3}\right)= & F_{3}\left(\mathbf{w}_{3}\right) E_{2}(u)-\sum_{t} \frac{F_{3}\left(\mathbf{w}_{3}-w_{t}\right)}{u-w_{t}}\left(F_{1}(u)-F_{1}\left(w_{t}\right)\right)- \\
& -\sum_{t \neq \hat{t}}\left(F_{4}(u)-F_{4}\left(w_{t}\right)\right) \frac{F_{3}\left(\mathbf{w}_{3}-w_{t}-w_{\hat{t}}\right)}{\left(u-w_{t}\right)\left(w_{t}-w_{\hat{t}}\right)} \\
& +\sum_{t \neq \hat{t}} \frac{F_{3}\left(\mathbf{w}_{3}-w_{t}\right)}{\left(u-w_{\hat{t}}\right)\left(w_{\hat{t}}-w_{t}\right)}-\sum_{t \hat{t}} \frac{F_{3}\left(\mathbf{w}_{3}+u-w_{t}-w_{t}\right)\left(w_{t}-w_{\hat{t}}\right)}{\left(u-w_{t}\right)} \\
E_{3}(u) F_{3}\left(\mathbf{w}_{3}\right)= & F_{3}\left(\mathbf{w}_{3}\right) E_{3}(u)-\sum_{1} \frac{F_{3}\left(\mathbf{w}_{3}-w_{t}\right)}{\left.u-E_{1}\left(w_{t}\right)\right)-} \\
& -\sum_{t \neq \hat{t}}\left(F_{2}(u)-F_{2}\left(w_{t}\right)\right) \frac{F_{3}\left(\mathbf{w}_{3}-w_{t}-w_{\hat{t}}\right)}{\left(u-w_{t}\right)\left(w_{t}-w_{\hat{t}}\right)}
\end{aligned}
$$

The similar formulas are valid for $F_{1}\left(\mathbf{w}_{\mathbf{1}}\right), F_{2}\left(\mathbf{w}_{\mathbf{2}}\right), F_{4}\left(\mathbf{w}_{\mathbf{4}}\right)$ but we will not write its explicitly here.

After this preparation we are able to calculate the action of $T(u)$

$$
T(u)\left|\mathbf{w}_{2}, \mathbf{w}_{3}, \mathbf{w}_{4}, \mathbf{w}_{1}\right\rangle=\left(T_{0}(u)+T_{1}(u)+T_{2}(u)+T_{3}(u)+T_{4}(u)\right)\left|\mathbf{w}_{2}, \mathbf{w}_{3}, \mathbf{w}_{4}, \mathbf{w}_{1}\right\rangle,
$$


The New Formulas for the Eigenvectors of the Gaudin Model in the so(5) Case.

where the final results for $T_{0}(u), T_{1}(u), T_{2}(u), T_{3}(u)$ and $T_{4}(u)$ are given in Appendix B.

Now we define

$$
\begin{aligned}
\mathbf{P}\left|\mathbf{w}_{2}, \mathbf{w}_{3}, \mathbf{w}_{4}, \mathbf{w}_{1}\right\rangle=\left(\sum_{a, d}\right. & \frac{\left|\mathbf{w}_{2}-w_{2, a}, \mathbf{w}_{3}+w_{2, a}, \mathbf{w}_{4}, \mathbf{w}_{1}-w_{1, d}\right\rangle}{w_{2, a}-w_{1, d}}+ \\
& \left.+\sum_{b, d} \frac{\left|\mathbf{w}_{2}, \mathbf{w}_{3}-w_{3, b}, \mathbf{w}_{4}+w_{3, b}, \mathbf{w}_{1}-w_{1, d}\right\rangle}{w_{3, b}-w_{1, d}}\right)
\end{aligned}
$$

Similarly as in [1] we define

$$
\left.\mid \mathbf{w}_{2}, \mathbf{w}_{1}\right)=\sum_{n=0}^{\infty} \frac{1}{n !} \mathbf{P}^{n}\left|\mathbf{w}_{2}, 0,0, \mathbf{w}_{1}\right\rangle .
$$

This is our ansatz for eigenvectors.

Theorem. If the Bethe Ansatz conditions: for any $d$

$$
\lambda_{2}\left(w_{1, d}\right)=\sum_{a} \frac{1}{w_{2, a}-w_{1, d}}+\sum_{d^{\prime} \neq d} \frac{1}{w_{1, d}-w_{1, d^{\prime}}}
$$

and for any $a$

$$
\lambda_{-}\left(w_{2, a}\right)=\sum_{a^{\prime} \neq a} \frac{2}{w_{2, a}-w_{2, a^{\prime}}}-\sum_{d} \frac{1}{w_{2, a}-w_{1, d}}
$$

are fulfilled then the vector

$$
\left.\mid \mathbf{w}_{2}, \mathbf{w}_{1}\right)=\sum_{n=0}^{\infty} \frac{\mathbf{P}^{n}}{n !}\left|\mathbf{w}_{2}, 0,0, \mathbf{w}_{1}\right\rangle
$$

is the eigenvector of the so(5) Gaudin model and

$$
\left.\left.T(u) \mid \mathbf{w}_{2}, \mathbf{w}_{1}\right)=\tau\left(u ; \mathbf{w}_{2}, \mathbf{w}_{1}\right) \mid \mathbf{w}_{2}, \mathbf{w}_{1}\right),
$$

where

$$
\begin{aligned}
\tau\left(u ; \mathbf{w}_{2}, \mathbf{w}_{1}\right)= & \tau(u)-\sum_{a}\left(\lambda_{-}(u)-\sum_{a^{\prime} \neq a} \frac{2}{w_{2, a}-w_{2, a^{\prime}}}+\sum_{d} \frac{1}{w_{2, a}-w_{1, d}}\right) \frac{1}{u-w_{2, a}}- \\
& -\sum_{d}\left(\lambda_{2}(u)+\sum_{a} \frac{1}{w_{1, d}-w_{2, a}}-\sum_{d^{\prime} \neq d} \frac{1}{w_{1, d}-w_{1, d^{\prime}}}\right) \frac{1}{u-w_{1, d}} \\
\tau(u)= & \frac{1}{2}\left(\lambda_{1}^{2}(u)+\lambda_{2}^{2}(u)-3 \lambda_{1}^{\prime}(u)-\lambda_{2}^{\prime}(u)\right) .
\end{aligned}
$$

Proof: In order to be $\left.\mid \mathbf{w}_{2}, \mathbf{w}_{1}\right)$ the eigenvector of the $T(u)$ with eigenvalue $\tau\left(u ; \mathbf{w}_{2}, \mathbf{w}_{1}\right)$, must be

$$
\begin{aligned}
& \left.\left.\left.\left.T_{1}(u) \mid \mathbf{w}_{2}, \mathbf{w}_{1}\right)=T_{2}(u) \mid \mathbf{w}_{2}, \mathbf{w}_{1}\right)=T_{3}(u) \mid \mathbf{w}_{2}, \mathbf{w}_{1}\right)=T_{4}(u) \mid \mathbf{w}_{2}, \mathbf{w}_{1}\right)=0 \\
& \left.\left.T_{0}(u) \mid \mathbf{w}_{2}, \mathbf{w}_{1}\right)=\tau\left(u ; \mathbf{w}_{2}, \mathbf{w}_{1}\right) \mid \mathbf{w}_{2}, \mathbf{w}_{1}\right) .
\end{aligned}
$$

In our paper we show $\left.T_{2}(u) \mid \mathbf{w}_{2}, \mathbf{w}_{1}\right)=0$ in the next Lemma 4 in the details. Because the proves of the relations $\left.\left.\left.T_{2}(u) \mid \mathbf{w}_{2}, \mathbf{w}_{1}\right)=T_{3}(u) \mid \mathbf{w}_{2}, \mathbf{w}_{1}\right)=T_{4}(u) \mid \mathbf{w}_{2}, \mathbf{w}_{1}\right)=0$ and $\left.\left.T_{0}(u) \mid \mathbf{w}_{2}, \mathbf{w}_{1}\right)=\tau\left(u ; \mathbf{w}_{2}, \mathbf{w}_{1}\right) \mid \mathbf{w}_{2}, \mathbf{w}_{1}\right)$ is similar we will skip it. 
The New Formulas for the Eigenvectors of the Gaudin Model in the so(5) Case.

Lemma 4. If it is valid (8) and (9) then $\left.T_{2}(u) \mid \mathbf{w}_{2}, \mathbf{w}_{1}\right)=0$.

Proof: From (11) in Appendix B we have

$$
\begin{aligned}
& T_{2}(u) \mid \mathbf{w}_{2},\left.\mathbf{w}_{3}, \mathbf{w}_{4}, \mathbf{w}_{1}\right\rangle= \\
&= \sum_{a}\left(\lambda_{-}\left(w_{2, a}\right)-\sum_{a^{\prime} \neq a} \frac{2}{w_{2, a}-w_{2, a^{\prime}}}-\sum_{b} \frac{1}{w_{2, a}-w_{3, b}}+\sum_{d} \frac{1}{w_{2, a}-w_{1, d}}\right) \times \\
& \quad \times \frac{\left|\mathbf{w}_{2}+u-w_{2, a}, \mathbf{w}_{3}, \mathbf{w}_{4}, \mathbf{w}_{1}\right\rangle}{u-w_{2, a}}- \\
& \quad-\sum_{a, b} \frac{\left|\mathbf{w}_{2}+u-w_{2, a}, \mathbf{w}_{3}+w_{2, a}-w_{3, b}, \mathbf{w}_{4}, \mathbf{w}_{1}\right\rangle}{\left(u-w_{3, b}\right)\left(w_{3, b}-w_{2, a}\right)}- \\
&-\sum_{a, d} \frac{\left|\mathbf{w}_{2}+u-w_{2, a}, \mathbf{w}_{3}, \mathbf{w}_{4}, \mathbf{w}_{1}+w_{2, a}-w_{1, d}\right\rangle}{\left(u-w_{2, a}\right)\left(w_{2, a}-w_{1, d}\right)}+ \\
&\left.+\sum_{b} \frac{\left|\mathbf{w}_{2}+u, \mathbf{w}_{3}-w_{3, b}, \mathbf{w}_{4}, \mathbf{w}_{1}+w_{3, b}\right\rangle}{u-w_{3, b}}-w_{3, b^{\prime}}, \mathbf{w}_{4}+w_{3, b}, \mathbf{w}_{1}\right\rangle \\
&-\sum_{b \neq b^{\prime}} \frac{\left.\mid \mathbf{w}_{2}+u, \mathbf{w}_{3}-w_{3, b}-w_{3, b^{\prime}}\right)\left(w_{3, b^{\prime}}-w_{3, b}\right)}{\left(u-w_{3}\right.}
\end{aligned}
$$

For the proof we define

$$
\begin{aligned}
\mathbf{P}_{1}\left|\mathbf{w}_{2}, \mathbf{w}_{3}, \mathbf{w}_{4}, \mathbf{w}_{1}\right\rangle & =\sum_{a, d} \frac{\left|\mathbf{w}_{2}-w_{2, a}, \mathbf{w}_{3}+w_{2, a}, \mathbf{w}_{4}, \mathbf{w}_{1}-w_{1, d}\right\rangle}{w_{2, a}-w_{1, d}} \\
\mathbf{P}_{2}\left|\mathbf{w}_{2}, \mathbf{w}_{3}, \mathbf{w}_{4}, \mathbf{w}_{1}\right\rangle & =\sum_{b, d} \frac{\left|\mathbf{w}_{2}, \mathbf{w}_{3}-w_{3, b}, \mathbf{w}_{4}+w_{3, b}, \mathbf{w}_{1}-w_{1, d}\right\rangle}{w_{3, b}-w_{1, d}} \\
\mathbf{P} & =\mathbf{P}_{1}+\mathbf{P}_{2} .
\end{aligned}
$$

By induction it is possible to prove that for any $n$ the relation

$$
\mathbf{P}^{n}\left|\mathbf{w}_{2}, 0,0, \mathbf{w}_{1}\right\rangle=\sum_{0 \leq k \leq n / 2} \frac{1}{2^{k}}\left(\begin{array}{l}
n \\
k
\end{array}\right) \mathbf{P}_{2}^{k} \mathbf{P}_{1}^{n-k}\left|\mathbf{w}_{2}, 0,0, \mathbf{w}_{1}\right\rangle
$$

hold and we can write

$$
\left.\mid \mathbf{w}_{2}, \mathbf{w}_{1}\right)=\sum_{n=0}^{\infty} \sum_{k=0}^{n} \frac{1}{2^{k} k ! n !} \mathbf{P}_{2}^{k} \mathbf{P}_{1}^{n}\left|\mathbf{w}_{2}, 0,0, \mathbf{w}_{1}\right\rangle .
$$

Further we point out that

$$
\mathbf{P}_{2}^{k} \mathbf{P}_{1}^{n}\left|\mathbf{w}_{2}, 0,0, \mathbf{w}_{1}\right\rangle=\frac{n !}{(n-k) !} \sum_{A_{n} ; D_{n+k}} \frac{\left|\mathbf{w}_{2}-A_{n}, A_{k, n}, A_{k}, \mathbf{w}_{1}-D_{n+k}\right\rangle}{X_{n} Y_{k, n}},
$$

where the summation is with respect all possible $A_{n}$ or $D_{n+k}$ ordered subsets of $\mathbf{w}_{2}$ or $\mathbf{w}_{1}, A_{k}$ and $A_{k, n}$ are the sets consist of the first $k$ and the last $n-k$ elements in $A_{n}$ and

$$
X_{n}=\prod_{r=1}^{n}\left(w_{2, a_{r}}-w_{1, d_{r}}\right), \quad Y_{k, n}=\prod_{r=1}^{k}\left(w_{2, a_{r}}-w_{1, d_{n+r}}\right) .
$$


The New Formulas for the Eigenvectors of the Gaudin Model in the so(5) Case.

Now we explicitly calculate in this notation

$$
\begin{aligned}
& T_{2}(u) \sum_{A_{n} ; D_{n+k}} \frac{\left|\mathbf{w}_{2}-A_{n}, A_{k, n}, A_{k}, \mathbf{w}_{1}-D_{n+k}\right\rangle}{X_{n} Y_{k, n}}= \\
& =\sum_{A_{n+1} ; D_{n+k}}\left(\lambda_{-}\left(w_{2, a_{n+1}}\right)-\sum_{a \notin A_{n+1}} \frac{2}{w_{2, a_{n+1}}-w_{2, a}}-\sum_{r=k+1}^{n} \frac{1}{w_{2, a_{n+1}}-w_{2, a_{r}}}+\right. \\
& \left.\quad+\sum_{d \notin D_{n+k}} \frac{1}{w_{2, a_{n+1}}-w_{1, d}}\right) \frac{\left|\mathbf{w}_{2}+u-A_{n+1}, A_{k, n}, A_{k}, \mathbf{w}_{1}-D_{n+k}\right\rangle}{\left(u-w_{2, a_{n+1}}\right) X_{n} Y_{k, n}}- \\
& -\sum_{A_{n+1} ; D_{n+k}} \sum_{A_{n+1} ; D_{n+k+1}}^{n} \frac{\left|\mathbf{w}_{2}+u-A_{n+1}, A_{k, n+1}-w_{2, a_{r}}, A_{k}, \mathbf{w}_{1}-D_{n+k}\right\rangle}{\left(u-w_{2, a_{r}}\right)\left(w_{2, a_{r}}-w_{2, a_{n+1}}\right) X_{n} Y_{k, n}}- \\
& +\sum_{A_{n} ; D_{n+k} r=k+1} \sum_{A_{n} ; D_{n+k} r \neq s=k+1}^{n} \frac{\left|\mathbf{w}_{2}+u-A_{n}, A_{k, n}-w_{2, a_{r}}, A_{k}, \mathbf{w}_{1}+w_{2, a_{r}}-D_{n+k}\right\rangle}{\left(u-w_{n+1}, A_{k, n}, A_{k}, \mathbf{w}_{1}+w_{2, a_{n+1}}-D_{n+k+1}\right\rangle}+ \\
& -\sum_{\left.A_{2, a_{n+1}}\right)\left(w_{2, a_{n+1}}-w_{1, a_{n+k}}\right) X_{n} Y_{k, n}} \sum_{k, n} \frac{\left|\mathbf{w}_{2}+u-A_{n}, A_{k, n}-w_{2, a_{r}}-w_{2, a_{s}}, A_{k}+w_{2, a_{r}}, \mathbf{w}_{1}-D_{n+k}\right\rangle}{\left(u-w_{2, a_{s}}\right)\left(w_{2, a_{s}}-w_{2, a_{r}}\right) X_{n} Y_{k, n}}
\end{aligned}
$$

If we substitute (9) and do some reordering of summation, we obtain

$$
\begin{aligned}
& T_{2}(u) \sum_{A_{n} ; D_{n+k}} \frac{\left|\mathbf{w}_{2}-A_{n}, A_{k, n}, A_{k}, \mathbf{w}_{1}-D_{n+k}\right\rangle}{X_{n} Y_{k, n}}= \\
& =\sum_{A_{n+1} ; D_{n+k}}\left(\sum_{r=1}^{k} \frac{2}{w_{2, a_{n+1}}-w_{2, a_{r}}}+\sum_{r=k+1}^{n} \frac{1}{w_{2, a_{n+1}}-w_{2, a_{r}}}-\sum_{r=1}^{n+k} \frac{1}{w_{2, a_{n+1}}-w_{1, d_{r}}}\right) \times \\
& \times \frac{\left|\mathbf{w}_{2}+u-A_{n+1}, A_{k, n}, A_{k}, \mathbf{w}_{1}-D_{n+k}\right\rangle}{\left(u-w_{2, a_{n+1}}\right) X_{n} Y_{k, n}}- \\
& -(n-k) \sum_{A_{n+1} ; D_{n+k}} \frac{\left|\mathbf{w}_{2}+u-A_{n+1}, A_{k+1, n+1}, A_{k}, \mathbf{w}_{1}-D_{n+k}\right\rangle}{\left(u-w_{2, a_{k+1}}\right)\left(w_{2, a_{k+1}}-w_{2, a_{n+1}}\right) X_{n} Y_{k, n}}- \\
& -\sum_{A_{n+1} ; D_{n+k+1}} \frac{\left|\mathbf{w}_{2}+u-A_{n+1}, A_{k, n}, A_{k}, \mathbf{w}_{1}+w_{2, a_{n+1}}-D_{n+k+1}\right\rangle}{\left(u-w_{2, a_{n+1}}\right)\left(w_{2, a_{n+1}}-w_{1, d_{n+k+1}}\right) X_{n} Y_{k, n}}+ \\
& +(n-k) \sum_{A_{n} ; D_{n+k}} \frac{\left|\mathbf{w}_{2}+u-A_{n}, A_{k, n-1}, A_{k}, \mathbf{w}_{1}+w_{2, a_{n}}-D_{n+k}\right\rangle}{\left(u-w_{2, a_{n}}\right) X_{n} Y_{k, n}}- \\
& -(n-k)(n-k-1) \sum_{A_{n} ; D_{n+k}} \frac{\left|\mathbf{w}_{2}+u-A_{n}, A_{k+1, n-1}, A_{k+1}, \mathbf{w}_{1}-D_{n+k}\right\rangle}{\left(u-w_{2, a_{n}}\right)\left(w_{2, a_{n}}-w_{2, a_{k+1}}\right) X_{n} Y_{k, n}}= \\
& =(n-k) \sum_{A_{n+1} ; D_{n+k}} \frac{\left|\mathbf{w}_{2}+u-A_{n+1}, A_{k, n}, A_{k}, \mathbf{w}_{1}-D_{n+k}\right\rangle}{\left(u-w_{2, a_{n+1}}\right)\left(w_{2, a_{n+1}}-w_{2, a_{n}}\right)\left(w_{2, a_{n+1}}-w_{1, d_{n}}\right) X_{n-1} Y_{k, n}}+ \\
& +k \sum_{A_{n+1} ; D_{n+k}} \frac{\left(w_{2, a_{k}}-w_{1, d_{k}}\right)\left|\mathbf{w}_{2}+u-A_{n+1}, A_{k, n}, A_{k}, \mathbf{w}_{1}-D_{n+k}\right\rangle}{\left(u-w_{2, a_{n+1}}\right)\left(w_{2, a_{n+1}}-w_{2, a_{k}}\right)\left(w_{2, a_{n+1}}-w_{1, d_{k}}\right) X_{n} Y_{k, n}}+ \\
& +k \sum_{A_{n+1} ; D_{n+k}} \frac{\left(w_{2, a_{k}}-w_{1, d_{n+k}}\right)\left|\mathbf{w}_{2}+u-A_{n+1}, A_{k, n}, A_{k}, \mathbf{w}_{1}-D_{n+k}\right\rangle}{\left(u-w_{2, a_{n+1}}\right)\left(w_{2, a_{n+1}}-w_{2, a_{k}}\right)\left(w_{2, a_{n+1}}-w_{1, d_{n+k}}\right) X_{n} Y_{k, n}}- \\
& -(n-k) \sum_{A_{n+1} ; D_{n+k}} \frac{\left(w_{2, a_{k+1}}-w_{1, d_{k+1}}\right)\left|\mathbf{w}_{2}+u-A_{n+1}, A_{k, n}, A_{k}, \mathbf{w}_{1}-D_{n+k}\right\rangle}{\left(u-w_{2, a_{n+1}}\right)\left(w_{2, a_{n+1}}-w_{2, a_{k+1}}\right)\left(w_{2, a_{n+1}}-w_{1, d_{k+1}}\right) X_{n} Y_{k, n}}- \\
& -(n-k)(n-k-1) \sum_{A_{n} ; D_{n+k}} \frac{\left|\mathbf{w}_{2}+u-A_{n}, A_{k+1, n-1}, A_{k+1}, \mathbf{w}_{1}-D_{n+k}\right\rangle}{\left(u-w_{2, a_{n}}\right)\left(w_{2, a_{n}}-w_{2, a_{k+1}}\right) X_{n} Y_{k, n}}-
\end{aligned}
$$


The New Formulas for the Eigenvectors of the Gaudin Model in the so(5) Case.

$$
\begin{aligned}
& -\sum_{A_{n+1} ; D_{n+k+1}} \frac{\left|\mathbf{w}_{2}+u-A_{n+1}, A_{k, n}, A_{k}, \mathbf{w}_{1}+w_{2, a_{n+1}}-D_{n+k+1}\right\rangle}{\left(u-w_{2, a_{n+1}}\right)\left(w_{2, a_{n+1}}-w_{1, d_{n+k+1}}\right) X_{n} Y_{k, n}}+ \\
& +(n-k) \sum_{A_{n} ; D_{n+k}} \frac{\left|\mathbf{w}_{2}+u-A_{n}, A_{k, n-1}, A_{k}, \mathbf{w}_{1}+w_{2, a_{n}}-D_{n+k}\right\rangle}{\left(u-w_{2, a_{n}}\right) X_{n} Y_{k, n}} .
\end{aligned}
$$

If we put this expression to (10) we obtain

$$
\begin{aligned}
& \left.T_{2}(u) \mid \mathbf{w}_{2}, \mathbf{w}_{1}\right)=\sum_{0 \leq k \leq n} \frac{1}{2^{k} k !(n-k) !} \times \\
& \times\left[\sum _ { A _ { n + 2 } ; D _ { n + k + 1 } } \left(\frac{\left|\mathbf{w}_{2}+u-A_{n+2}, A_{k, n+1}, A_{k}, \mathbf{w}_{1}-D_{n+k+1}\right\rangle}{\left(u-w_{2, a_{n+2}}\right)\left(w_{2, a_{n+2}}-w_{2, a_{n+1}}\right)\left(w_{2, a_{n+2}}-w_{1, d_{n+1}}\right) X_{n} Y_{k, n+1}}-\right.\right. \\
& \left.-\frac{\left(w_{2, a_{k+1}}-w_{1, d_{k+1}}\right)\left|\mathbf{w}_{2}+u-A_{n+2}, A_{k, n+1}, A_{k}, \mathbf{w}_{1}-D_{n+k+1}\right\rangle}{\left(u-w_{2, a_{n+2}}\right)\left(w_{2, a_{n+2}}-w_{2, a_{k+1}}\right)\left(w_{2, a_{n+2}}-w_{1, d_{k+1}}\right) X_{n+1} Y_{k, n+1}}\right)+ \\
& +\sum_{A_{n+2} ; D_{n+k+2}}\left(\frac{1}{2} \frac{\left(w_{2, a_{k+1}}-w_{1, d_{k+1}}\right)\left|\mathbf{w}_{2}+u-A_{n+2}, A_{k+1, n+1}, A_{k+1}, \mathbf{w}_{1}-D_{n+k+2}\right\rangle}{\left(u-w_{2, a_{n+2}}\right)\left(w_{2, a_{n+2}}-w_{2, a_{k+1}}\right)\left(w_{2, a_{n+2}}-w_{1, d_{k+1}}\right) X_{n+1} Y_{k+1, n+1}}+\right. \\
& +\frac{1}{2} \frac{\left(w_{2, a_{k+1}}-w_{1, d_{n+k+2}}\right)\left|\mathbf{w}_{2}+u-A_{n+2}, A_{k+1, n+1}, A_{k+1}, \mathbf{w}_{1}-D_{n+k+2}\right\rangle}{\left(u-w_{2, a_{n+2}}\right)\left(w_{2, a_{n+2}}-w_{2, a_{k+1}}\right)\left(w_{2, a_{n+2}}-w_{1, d_{n+k+2}}\right) X_{n+1} Y_{k+1, n+1}}- \\
& \left.-\frac{\left|\mathbf{w}_{2}+u-A_{n+2}, A_{k+1, n+1}, A_{k+1}, \mathbf{w}_{1}-D_{n+k+2}\right\rangle}{\left(u-w_{2, a_{n+2}}\right)\left(w_{2, a_{n+2}}-w_{2, a_{k+1}}\right) X_{n+2} Y_{k, n+2}}\right)+ \\
& +\sum_{A_{n+1} ; D_{n+k+1}}\left(\frac{\left|\mathbf{w}_{2}+u-A_{n+1}, A_{k, n}, A_{k}, \mathbf{w}_{1}+w_{2, a_{n+1}}-D_{n+k+1}\right\rangle}{\left(u-w_{2, a_{n+1}}\right) X_{n+1} Y_{k, n+1}}-\right. \\
& \left.\left.-\frac{\left|\mathbf{w}_{2}+u-A_{n+1}, A_{k, n}, A_{k}, \mathbf{w}_{1}+w_{2, a_{n+1}}-D_{n+k+1}\right\rangle}{\left(u-w_{2, a_{n+1}}\right)\left(w_{2, a_{n+1}}-w_{1, d_{n+k+1}}\right) X_{n} Y_{k, n}}\right)\right]
\end{aligned}
$$

If we use substitution $\left(a_{k+1}, d_{k+1}\right) \leftrightarrow\left(a_{n+1}, d_{n+1}\right)$ the first member will be cancelled.

We see that last term is zero too after the transformation $d_{n+1} \rightarrow d_{n+2} \rightarrow \ldots \rightarrow$ $d_{n+k+1} \rightarrow d_{n+1}$.

The second term have two constituents. The substitution $d_{k+1} \leftrightarrow d_{n+k+2}$ transforms first on second

$$
\begin{aligned}
& \sum_{A_{n+2} ; D_{n+k+2}}\left(\frac{1}{\left(w_{2, a_{n+2}}-w_{1, d_{n+k+2}}\right) X_{n+1} Y_{k, n+1}}-\frac{1}{X_{n+2} Y_{k, n+2}}\right) \times \\
& \times \frac{\left|\mathbf{w}_{2}+u-A_{n+2}, A_{k+1, n+1}, A_{k+1}, \mathbf{w}_{1}-D_{n+k+2}\right\rangle}{\left(u-w_{2, a_{n+2}}\right)\left(w_{2, a_{n+2}}-w_{2, a_{k+1}}\right)}
\end{aligned}
$$

so after the substitution $d_{n+2} \rightarrow d_{n+3} \rightarrow \ldots \rightarrow d_{n+k+2} \rightarrow d_{n+2}$ this will be cancelled.

\section{Concluding remarks and open problems}

In the present paper we have proposed the new formulas for the eigenvalues of the Gaudin model obtained by using the Bethe ansatz method in so(5) case.

In the nineties last century Feigin, Frenkel, Reshetikhin [15] proved the formulas for eigenvectors for general semisimple Lie algebra $g$. The eigenvectors should be constructed by applying to the vacuum $|0\rangle$, the operators $F_{j}(w), \mathrm{j}=1, \ldots, \mathrm{l}$, connected 
with simple roots. If now $\left.F_{1}\left(w_{1}\right)\right)$ and $\left.F_{2}\left(w_{2}\right)\right)$ not commute we are not able to find Bethe equations for $\left.\left.F_{1}\left(w_{1}\right)\right) F_{2}\left(w_{2}\right)\right)|0\rangle$. So it is needed to add some extra terms. The right formula can be extracted from solutions of the $\mathrm{KZ}$ equation [16](and in fact can be obtained as quai-classical asymptotic of such solutions [17].)

$$
\left|w_{1}^{i_{1}}, w_{2}^{i_{2}}, \ldots, w_{m}^{i_{m}}\right\rangle=\sum_{p=\left\{I^{1}, \ldots, I^{N}\right\}} \prod_{j=1}^{N} \frac{f_{i_{1}^{j}}^{(j)} f_{i_{2}^{j}}^{(j)} \ldots f_{i_{a}^{j}}^{(j)}}{\left(w_{i_{1}^{j}}-w_{i_{2}^{j}}\right)\left(w_{i_{2}^{j}}-w_{i_{3}^{j}}\right) \ldots\left(w_{i_{a}^{j}}-z_{j}\right)}|0\rangle .
$$

Here the summation is taken over all ordered partitions $I^{1} \cup I^{2} \cup \ldots \cup I^{N}$ of the set $\{1, \ldots, m\}$, where $I^{j}=\left\{i_{1}^{j}, i_{2}^{j}, \ldots, i_{a_{j}}^{j}\right\}$. The proof is based on Wakimoto modules over affine algebras at the critical level [15].

The first interesting problem is to find explicit connection. We were able to rewrite one to other only in some simple examples as:

$$
\left|\mathbf{w}_{2,1}, \mathbf{w}_{1,1}\right\rangle=\left(F_{1}\left(w_{1}\right) F_{2}\left(w_{2}\right)+\frac{F_{3}\left(w_{1}\right)}{w_{1}-w_{2}}\right)|0\rangle
$$

and

$$
\left|\mathbf{w}_{2,1}, \mathbf{w}_{2,2}, \mathbf{w}_{1,1}, \mathbf{w}_{1,2}\right\rangle=\text { spočítam!!!!!!! }
$$

So it is look as an interesting question to find general connection.

In our paper [1] we study the case of algebra sl(3) explicitly and here the so(5) case. We believe that similar formulas are possible for general semisimple Lie algebra. Some calculation for other algebras in progress. So the second open problem is to generalize our results for other Lie algebras.

All proofs in presented paper are direct calculations. So as the last problem we formulate is to find some indirect proof which can be useful in general case.

\section{Acknowledgments}

This work was partially supported by research plan MSM6840770039.

\section{Appendix A}

We will work in basis $\mathbf{h}_{1}, \mathbf{h}_{2}, \mathbf{e}_{k}$ and $\mathbf{f}_{k}, k=1, \ldots, 4$, and use explicit commutation relations

$$
\begin{array}{llll}
{\left[\mathbf{h}_{1}, \mathbf{e}_{1}\right]=0,} & {\left[\mathbf{h}_{1}, \mathbf{e}_{2}\right]=\mathbf{e}_{2},} & {\left[\mathbf{h}_{1}, \mathbf{e}_{3}\right]=\mathbf{e}_{3},} & {\left[\mathbf{h}_{1}, \mathbf{e}_{4}\right]=\mathbf{e}_{4},} \\
{\left[\mathbf{h}_{2}, \mathbf{e}_{1}\right]=\mathbf{e}_{1},} & {\left[\mathbf{h}_{2}, \mathbf{e}_{2}\right]=-\mathbf{e}_{2},} & {\left[\mathbf{h}_{2}, \mathbf{e}_{3}\right]=0,} & {\left[\mathbf{h}_{2}, \mathbf{e}_{4}\right]=\mathbf{e}_{4}} \\
{\left[\mathbf{h}_{1}, \mathbf{f}_{1}\right]=0,} & {\left[\mathbf{h}_{1}, \mathbf{f}_{2}\right]=-\mathbf{f}_{2},} & {\left[\mathbf{h}_{1}, \mathbf{f}_{3}\right]=-\mathbf{f}_{3},} & {\left[\mathbf{h}_{1}, \mathbf{f}_{4}\right]=-\mathbf{f}_{4},} \\
{\left[\mathbf{h}_{2}, \mathbf{f}_{1}\right]=-\mathbf{f}_{1},} & {\left[\mathbf{h}_{2}, \mathbf{f}_{2}\right]=\mathbf{f}_{2},} & {\left[\mathbf{h}_{2}, \mathbf{f}_{3}\right]=0,} & {\left[\mathbf{h}_{2}, \mathbf{f}_{4}\right]=-\mathbf{f}_{4}} \\
{\left[\mathbf{e}_{1}, \mathbf{e}_{2}\right]=\mathbf{e}_{3},} & {\left[\mathbf{e}_{1}, \mathbf{e}_{3}\right]=\mathbf{e}_{4},} & {\left[\mathbf{e}_{1}, \mathbf{e}_{4}\right]=0,} & \\
{\left[\mathbf{e}_{2}, \mathbf{e}_{3}\right]=0,} & {\left[\mathbf{e}_{2}, \mathbf{e}_{4}\right]=0,} & {\left[\mathbf{e}_{3}, \mathbf{e}_{4}\right]=0,} &
\end{array}
$$


The New Formulas for the Eigenvectors of the Gaudin Model in the so(5) Case.

$$
\begin{aligned}
& {\left[\mathbf{f}_{1}, \mathbf{f}_{2}\right]=-\mathbf{f}_{3}, \quad\left[\mathbf{f}_{1}, \mathbf{f}_{3}\right]=-\mathbf{f}_{4}, \quad\left[\mathbf{f}_{1}, \mathbf{f}_{4}\right]=0,} \\
& {\left[\mathbf{f}_{2}, \mathbf{f}_{3}\right]=0, \quad\left[\mathbf{f}_{2}, \mathbf{f}_{4}\right]=0, \quad\left[\mathbf{f}_{3}, \mathbf{f}_{4}\right]=0,} \\
& {\left[\mathbf{e}_{1}, \mathbf{f}_{1}\right]=\mathbf{h}_{2}, \quad\left[\mathbf{e}_{1}, \mathbf{f}_{2}\right]=0, \quad\left[\mathbf{e}_{1}, \mathbf{f}_{3}\right]=-\mathbf{f}_{2}, \quad\left[\mathbf{e}_{1}, \mathbf{f}_{4}\right]=-\mathbf{f}_{3}} \\
& {\left[\mathbf{e}_{2}, \mathbf{f}_{1}\right]=0, \quad\left[\mathbf{e}_{2}, \mathbf{f}_{2}\right]=\mathbf{h}_{1}-\mathbf{h}_{2}, \quad\left[\mathbf{e}_{2}, \mathbf{f}_{3}\right]=\mathbf{f}_{1}, \quad\left[\mathbf{e}_{2}, \mathbf{f}_{4}\right]=0} \\
& {\left[\mathbf{e}_{3}, \mathbf{f}_{1}\right]=-\mathbf{e}_{2}, \quad\left[\mathbf{e}_{3}, \mathbf{f}_{2}\right]=\mathbf{e}_{1}, \quad\left[\mathbf{e}_{3}, \mathbf{f}_{3}\right]=\mathbf{h}_{1}, \quad\left[\mathbf{e}_{3}, \mathbf{f}_{4}\right]=\mathbf{f}_{1}} \\
& {\left[\mathbf{e}_{4}, \mathbf{f}_{1}\right]=-\mathbf{e}_{3}, \quad\left[\mathbf{e}_{4}, \mathbf{f}_{2}\right]=0, \quad\left[\mathbf{e}_{4}, \mathbf{f}_{3}\right]=\mathbf{e}_{1}, \quad\left[\mathbf{e}_{4}, \mathbf{f}_{4}\right]=\mathbf{h}_{1}+\mathbf{h}_{2}}
\end{aligned}
$$

\section{Appendix B}

After a lengthy and troublesome calculation we obtain for action of $T(u)$ )

$$
T(u)\left|\mathbf{w}_{2}, \mathbf{w}_{3}, \mathbf{w}_{4}, \mathbf{w}_{1}\right\rangle=\left(T_{0}(u)+T_{1}(u)+T_{2}(u)+T_{3}(u)+T_{4}(u)\right)\left|\mathbf{w}_{2}, \mathbf{w}_{3}, \mathbf{w}_{4}, \mathbf{w}_{1}\right\rangle,
$$

where

$$
\begin{aligned}
& T_{1}(u)\left|\mathbf{w}_{2}, \mathbf{w}_{3}, \mathbf{w}_{4}, \mathbf{w}_{1}\right\rangle= \\
& =\sum_{d}\left(\lambda_{2}\left(w_{1, d}\right)+\sum_{a} \frac{1}{u-w_{2, a}}-\sum_{c} \frac{1}{u-w_{4, c}}-\sum_{d^{\prime} \neq d} \frac{1}{w_{1, d}-w_{1, d^{\prime}}}\right) \times \\
& \quad \times \frac{\left|\mathbf{w}_{2}, \mathbf{w}_{3}, \mathbf{w}_{4}, \mathbf{w}_{1}+u-w_{1, d}\right\rangle}{u-w_{1, d}}+ \\
& \left.\quad+\sum_{a ; d \neq d^{\prime}} \frac{\left|\mathbf{w}_{2}-w_{2, a}, \mathbf{w}_{3}+w_{2, a}, \mathbf{w}_{4}, \mathbf{w}_{1}+u-w_{1, d}-w_{1, d^{\prime}}\right\rangle}{\left(u-w_{2, a}\right)\left(u-w_{1, d}\right)\left(w_{1, d}-w_{1, d^{\prime}}\right)}+w_{1, d^{\prime}}\right\rangle \\
& \quad+\sum_{b ; d \neq d^{\prime}} \frac{\left.\mid \mathbf{w}_{2}, \mathbf{w}_{3}-w_{3, b}, \mathbf{w}_{4}+w_{3, b}, \mathbf{w}_{1}+u-w_{1, d}-w_{1, d^{\prime}}\right)}{\left(u-w_{3, b}\right)\left(u-w_{1, d}\right)\left(w_{1, d}-w_{1}\right.}- \\
& \quad-\sum_{b} \frac{\left|\mathbf{w}_{2}+w_{3, b}, \mathbf{w}_{3}-w_{3, b}, \mathbf{w}_{4}, \mathbf{w}_{1}+u\right\rangle}{u-w_{3, b}}- \\
& \quad-\sum_{c} \frac{\left|\mathbf{w}_{2}, \mathbf{w}_{3}+w_{4, c}, \mathbf{w}_{4}-w_{4, c}, \mathbf{w}_{1}+u\right\rangle}{u-w_{4, c}}
\end{aligned}
$$

$$
\begin{aligned}
& T_{2}(u)\left|\mathbf{w}_{2}, \mathbf{w}_{3}, \mathbf{w}_{4}, \mathbf{w}_{1}\right\rangle= \\
& =\sum_{a}\left(\lambda_{-}\left(w_{2, a}\right)-\sum_{a^{\prime} \neq a} \frac{2}{w_{2, a}-w_{2, a^{\prime}}}-\sum_{b} \frac{1}{w_{2, a}-w_{3, b}}+\sum_{d} \frac{1}{w_{2, a}-w_{1, d}}\right) \times \\
& \quad \times \frac{\left|\mathbf{w}_{2}+u-w_{2, a}, \mathbf{w}_{3}, \mathbf{w}_{4}, \mathbf{w}_{1}\right\rangle}{u-w_{2, a}}- \\
& \quad-\sum_{a, b} \frac{\left|\mathbf{w}_{2}+u-w_{2, a}, \mathbf{w}_{3}+w_{2, a}-w_{3, b}, \mathbf{w}_{4}, \mathbf{w}_{1}\right\rangle}{\left(u-w_{3, b}\right)\left(w_{3, b}-w_{2, a}\right)}- \\
& \quad-\sum_{a, d} \frac{\left|\mathbf{w}_{2}+u-w_{2, a}, \mathbf{w}_{3}, \mathbf{w}_{4}, \mathbf{w}_{1}+w_{2, a}-w_{1, d}\right\rangle}{\left(u-w_{2, a}\right)\left(w_{2, a}-w_{1, d}\right)}+ \\
& \quad+\sum_{b} \frac{\left|\mathbf{w}_{2}+u, \mathbf{w}_{3}-w_{3, b}, \mathbf{w}_{4}, \mathbf{w}_{1}+w_{3, b}\right\rangle}{u-w_{3, b}}- \\
& \quad-\sum_{b \neq b^{\prime}} \frac{\left|\mathbf{w}_{2}+u, \mathbf{w}_{3}-w_{3, b}-w_{3, b^{\prime}}, \mathbf{w}_{4}+w_{3, b}, \mathbf{w}_{1}\right\rangle}{\left(u-w_{3, b^{\prime}}\right)\left(w_{3, b^{\prime}}-w_{3, b}\right)}
\end{aligned}
$$


$T_{3}(u)\left|\mathbf{w}_{2}, \mathbf{w}_{3}, \mathbf{w}_{4}, \mathbf{w}_{1}\right\rangle=$

$$
\begin{aligned}
= & \sum_{b}\left(\lambda_{1}\left(w_{3, b}\right)-\sum_{a} \frac{1}{w_{3, b}-w_{2, a}}-\sum_{b^{\prime} \neq b} \frac{1}{w_{3, b}-w_{3, b^{\prime}}}-\sum_{c} \frac{1}{w_{3, b}-w_{4, c}}\right) \times \\
& \times \frac{\left|\mathbf{w}_{2}, \mathbf{w}_{3}+u-w_{3, b}, \mathbf{w}_{4}, \mathbf{w}_{1}\right\rangle}{u-w_{3, b}}- \\
& -\sum_{a, b} \frac{\left|\mathbf{w}_{2}+w_{3, b}-w_{2, a}, \mathbf{w}_{3}+u-w_{3, b}, \mathbf{w}_{4}, \mathbf{w}_{1}\right\rangle}{\left(u-w_{2, a}\right)\left(w_{2, a}-w_{3, b}\right)}- \\
& -\sum_{a, c} \frac{\left|\mathbf{w}_{2}-w_{2, a}, \mathbf{w}_{3}+u+w_{4, c}, \mathbf{w}_{4}-w_{4, c}, \mathbf{w}_{1}\right\rangle}{\left(u-w_{2, a}\right)\left(w_{2, a}-w_{4, c}\right)}- \\
& -\sum_{a, c} \frac{\left|\mathbf{w}_{2}-w_{2, a}, \mathbf{w}_{3}+u+w_{2, a}, \mathbf{w}_{4}-w_{4, c}, \mathbf{w}_{1}\right\rangle}{\left(u-w_{4, c}\right)\left(w_{4, c}-w_{2, a}\right)}- \\
& -\sum_{b, c} \frac{\left|\mathbf{w}_{2}, \mathbf{w}_{3}+u-w_{3, b}, \mathbf{w}_{4}+w_{3, b}-w_{4, c}, \mathbf{w}_{1}\right\rangle}{\left(u-w_{4, c}\right)\left(w_{4, c}-w_{3, b}\right)}- \\
& -\sum_{a, d}\left(\lambda_{2}\left(w_{2, a}\right)-\lambda_{2}\left(w_{1, d}\right)\right) \frac{\left|\mathbf{w}_{2}-w_{2, a}, \mathbf{w}_{3}+u, \mathbf{w}_{4}, \mathbf{w}_{1}-w_{1, d}\right\rangle}{\left(u-w_{2, a}\right)\left(w_{2, a}-w_{1, d}\right)}+ \\
& +\sum_{a ; d \neq d^{\prime}} \frac{\left|\mathbf{w}_{2}-w_{2, a}, \mathbf{w}_{3}+u, \mathbf{w}_{4}, \mathbf{w}_{1}-w_{1, d}\right\rangle}{\left(u-w_{2, a}\right)\left(w_{2, a}-w_{1, d^{\prime}}\right)\left(w_{1, d^{\prime}}-w_{1, d}\right)}- \\
& -\sum_{a ; d \neq d^{\prime}} \frac{\left|\mathbf{w}_{2}-w_{2, a}, \mathbf{w}_{3}+u, \mathbf{w}_{4}, \mathbf{w}_{1}+w_{2, a}-w_{1, d}-w_{1, d^{\prime}}\right\rangle}{\left(u-w_{2, a}\right)\left(w_{2, a}-w_{1, d}\right)\left(w_{1, d}-w_{1, d^{\prime}}\right)}+ \\
& +\sum_{c} \frac{\left|\mathbf{w}_{2}, \mathbf{w}_{3}+u, \mathbf{w}_{4}-w_{4, c}, \mathbf{w}_{1}+w_{4, c}\right\rangle}{u-w_{4, c}}
\end{aligned}
$$

$T_{4}(u)\left|\mathbf{w}_{2}, \mathbf{w}_{3}, \mathbf{w}_{4}, \mathbf{w}_{1}\right\rangle=$

$$
\begin{gathered}
=\sum_{c}\left(\lambda_{+}\left(w_{4, c}\right)-\sum_{b} \frac{1}{w_{4, c}-w_{3, b}}-\sum_{c^{\prime} \neq c} \frac{2}{w_{4, c}-w_{4, c^{\prime}}}-\sum_{d} \frac{1}{w_{4, c}-w_{1, d}}\right) \times \\
\quad \times \frac{\left|\mathbf{w}_{2}, \mathbf{w}_{3}, \mathbf{w}_{4}+u-w_{4, c}, \mathbf{w}_{1}\right\rangle}{u-w_{4, c}}- \\
-\sum_{b, d}\left(\lambda_{2}\left(w_{3, b}\right)-\lambda_{2}\left(w_{1, d}\right)\right) \frac{\left|\mathbf{w}_{2}, \mathbf{w}_{3}-w_{3, b}, \mathbf{w}_{4}+u, \mathbf{w}_{1}-w_{1, d}\right\rangle}{\left(u-w_{3, b}\right)\left(w_{3, b}-w_{1, d}\right)}+ \\
+\sum_{b ; d \neq d^{\prime}} \frac{\left|\mathbf{w}_{2}, \mathbf{w}_{3}-w_{3, b}, \mathbf{w}_{4}+u, \mathbf{w}_{1}-w_{1, d}\right\rangle}{\left(u-w_{3, b}\right)\left(w_{3, b}-w_{1, d^{\prime}}\right)\left(w_{1, d^{\prime}}-w_{1, d}\right)}- \\
-\sum_{b ; d \neq d^{\prime}} \frac{\left|\mathbf{w}_{2}, \mathbf{w}_{3}-w_{3, b}, \mathbf{w}_{4}+u, \mathbf{w}_{1}+w_{3, b}-w_{1, d}-w_{1, d^{\prime}}\right\rangle}{\left(u-w_{3, b}\right)\left(w_{3, b}-w_{1, d}\right)\left(w_{1, d}-w_{1, d^{\prime}}\right)}- \\
-\sum_{b, c} \frac{\left|\mathbf{w}_{2}, \mathbf{w}_{3}+w_{4, c}-w_{3, b}, \mathbf{w}_{4}+u-w_{4, c} \mathbf{w}_{1}\right\rangle}{\left(u-w_{3, b}\right)\left(w_{3, b}-w_{4, c}\right)}- \\
-\sum_{b \neq b^{\prime}} \frac{\left|\mathbf{w}_{2}+w_{3, b}, \mathbf{w}_{3}-w_{3, b}-w_{3, b^{\prime}}, \mathbf{w}_{4}+u, \mathbf{w}_{1}\right\rangle}{\left(u-w_{3, b^{\prime}}\right)\left(w_{3, b^{\prime}}-w_{3, b}\right)}+ \\
+\sum_{c, d} \frac{\left|\mathbf{w}_{2}, \mathbf{w}_{3}, \mathbf{w}_{4}+u-w_{4, c}, \mathbf{w}_{1}+w_{4, c}-w_{1, d}\right\rangle}{\left(u-w_{4, c}\right)\left(w_{4, c}-w_{1, d}\right)}
\end{gathered}
$$


The New Formulas for the Eigenvectors of the Gaudin Model in the so(5) Case.

$$
\begin{aligned}
& T_{0}(u)\left|\mathbf{w}_{2}, \mathbf{w}_{3}, \mathbf{w}_{4}, \mathbf{w}_{1}\right\rangle=\tau(u)\left|\mathbf{w}_{2}, \mathbf{w}_{3}, \mathbf{w}_{4}, \mathbf{w}_{1}\right\rangle- \\
& -\sum_{a}\left(\lambda_{-}(u)-\sum_{a^{\prime} \neq a} \frac{2}{w_{2, a}-w_{2, a^{\prime}}}-\sum_{b} \frac{1}{w_{2, a}-w_{3, b}}+\sum_{d} \frac{1}{w_{2, a}-w_{1, d}}\right) \times \\
& \times \frac{\left|\mathbf{w}_{2}, \mathbf{w}_{3}, \mathbf{w}_{4}, \mathbf{w}_{1}\right\rangle}{u-w_{2, a}}- \\
& -\sum_{b}\left(\lambda_{1}(u)-\sum_{a} \frac{1}{w_{3, b}-w_{2, a}}-\sum_{b^{\prime} \neq b} \frac{1}{w_{3, b}-w_{3, b^{\prime}}}-\sum_{c} \frac{1}{w_{3, b}-w_{4, c}}\right) \times \\
& \times \frac{\left|\mathbf{w}_{2}, \mathbf{w}_{3}, \mathbf{w}_{4}, \mathbf{w}_{1}\right\rangle}{u-w_{3, b}}- \\
& -\sum_{c}\left(\lambda_{+}(u)-\sum_{b} \frac{1}{w_{4, c}-w_{3, b}}-\sum_{c^{\prime} \neq c} \frac{2}{w_{4, c}-w_{4, c^{\prime}}}-\sum_{d} \frac{1}{w_{4, c}-w_{1, d}}\right) \times \\
& \times \frac{\left|\mathbf{w}_{2}, \mathbf{w}_{3}, \mathbf{w}_{4}, \mathbf{w}_{1}\right\rangle}{u-w_{3, c}}- \\
& -\sum_{d}\left(\lambda_{2}(u)+\sum_{a} \frac{1}{w_{1, d}-w_{2, a}}-\sum_{c} \frac{1}{w_{1, d}-w_{4, c}}-\sum_{d^{\prime} \neq d} \frac{1}{w_{1, d}-w_{1, d^{\prime}}}\right) \times \\
& \times \frac{\left|\mathbf{w}_{2}, \mathbf{w}_{3}, \mathbf{w}_{\mathbf{4}}, \mathbf{w}_{1}\right\rangle}{u-w_{1, d}}+ \\
& +\sum_{a, d}\left(\lambda_{2}(u)-\lambda_{2}\left(w_{1, d}\right)\right) \frac{\left|\mathbf{w}_{2}-w_{2, a}, \mathbf{w}_{3}+w_{2, a}, \mathbf{w}_{4}, \mathbf{w}_{1}-w_{1, d}\right\rangle}{\left(u-w_{2, a}\right)\left(u-w_{1, d}\right)}- \\
& -\sum_{a ; d \neq d^{\prime}} \frac{\left|\mathbf{w}_{2}-w_{2, a}, \mathbf{w}_{3}+w_{2, a}, \mathbf{w}_{4}, \mathbf{w}_{1}-w_{1, d}\right\rangle}{\left(u-w_{2, a}\right)\left(u-w_{1, d^{\prime}}\right)\left(w_{1, d^{\prime}}-w_{1, d}\right)}+ \\
& +\sum_{b, d}\left(\lambda_{2}(u)-\lambda_{2}\left(w_{1, d}\right)\right) \frac{\left|\mathbf{w}_{2}, \mathbf{w}_{3}-w_{3, b}, \mathbf{w}_{4}+w_{3, b}, \mathbf{w}_{1}-w_{1, d}\right\rangle}{\left(u-w_{3, b}\right)\left(u-w_{1, d}\right)}- \\
& -\sum_{b ; d \neq d^{\prime}} \frac{\left|\mathbf{w}_{2}, \mathbf{w}_{3}-w_{3, b}, \mathbf{w}_{4}+w_{3, b}, \mathbf{w}_{1}-w_{1, d}\right\rangle}{\left(u-w_{3, b}\right)\left(u-w_{1, d^{\prime}}\right)\left(w_{1, d^{\prime}}-w_{1, d}\right)}+ \\
& +\sum_{a, b} \frac{\left|\mathbf{w}_{2}+w_{3, b}-w_{2, a}, \mathbf{w}_{3}+w_{2, a}-w_{3, b}, \mathbf{w}_{4}, \mathbf{w}_{1}\right\rangle}{\left(u-w_{2, a}\right)\left(u-w_{3, b}\right)}+ \\
& +\sum_{a, c} \frac{\left|\mathbf{w}_{2}-w_{2, a}, \mathbf{w}_{3}+w_{2, a}+w_{4, c}, \mathbf{w}_{4}-w_{4, c}, \mathbf{w}_{1}\right\rangle}{\left(u-w_{2, a}\right)\left(u-w_{4, c}\right)}+ \\
& +\sum_{b, c} \frac{\left|\mathbf{w}_{2}, \mathbf{w}_{3}+w_{4, c}-w_{3, b}, \mathbf{w}_{4}+w_{3, b}-w_{4, c}, \mathbf{w}_{1}\right\rangle}{\left(u-w_{3, b}\right)\left(u-w_{4, c}\right)}+ \\
& +\sum_{b \neq b^{\prime}} \frac{\left|\mathbf{w}_{2}+w_{3, b}, \mathbf{w}_{3}-w_{3, b}-w_{3, b^{\prime}}, \mathbf{w}_{4}+w_{3, b^{\prime}}, \mathbf{w}_{1}\right\rangle}{\left(u-w_{3, b}\right)\left(u-w_{3, b^{\prime}}\right)}
\end{aligned}
$$

\section{References}

[1] Burdík Č and Navrátil O 2008 Regular and Chaotic Dynamic 13403

[2] Gaudin M 1972 Note CEA 1559, CEN-Saclay. Nevím, co s tou citací????????

[3] Gaudin M 1976 J. Physique. 371087

[4] Gaudin M 1983 La Fonction d'Onde de Bethe, Masson, Paris

[5] Kalnins E G, Kuznetsov V B and Miller W Jr 1994 J. Math. Phys. 35 1710-1731, http://arxiv.org/abs/hep-th/9308109hep-th/9308109

[6] Musso F, Petrera M and Ragnisco O 2005 J. Nonlinear Math. Phys. 12 482, http://arxiv.org/abs/nlin.SI/0410016nlin.SI/0410016. 
[7] Petrera M 2007 Integrable extensions and discretizations of classical Gaudin models, $\mathrm{PhD}$ Thesis Dipartimento di Fisica E. Amaldi, Università degli Studi Roma Tre, Rome, Italy.

[8] Reyman A G and Semenov-Tian-Shansky M A 1994 in Dynamical Systems VII, Editors V.I. Arnold and S.P. Novikov, Encyclopaedia of Mathematical Sciences, Vol. 16, Berlin, Springer, 116

[9] Sklyanin E K 1987 in Differentsialnaya Geom. Gruppy Li i Mekh. IX, Zap. Nauchn. Sem. Leningrad. Otdel. Mat. Inst. Steklov. (LOMI) 164 151; (English transl. 1989 J. Soviet Math. 47 2473)

[10] Sklyanin E K 1987 in Vopr. Kvant. Teor. Polya i Statist. Fiz. 7, Zap. Nauchn. Sem. Leningrad. Otdel. Mat. Inst. Steklov. (LOMI) 161 88, 176, 179; (English transl. 1989 J. Soviet Math. 46 2104)

[11] Hone A N W, Kuznetsov V B and Ragnisco O 2001 J. Phys. A: Math. Gen. 34 2477, http://arxiv.org/abs/nlin.SI/0007041nlin.SI/0007041.

[12] Baxter R 1982 Exacty solved models in statistical mechanics, New York: Academic Press

[13] Faddeev L and Takhtajan L 1976 Russ. Math. Surv. 3411

[14] Sklyanin E 1992 Nankai Lectures in Mathematical Physics, ed. Mo-Lin Ge, Singapore: World Scientific, 63

[15] Feigin B, Frenkel E and Reshetikhin N 1994 Commun. Math. Phys. 16627

[16] Schechtman V and Varchenko A 1991 Invent. Math. 106680

[17] Reshetikhin N and Varchenko A 1994 arXiv:hep-th/9402126v3. 